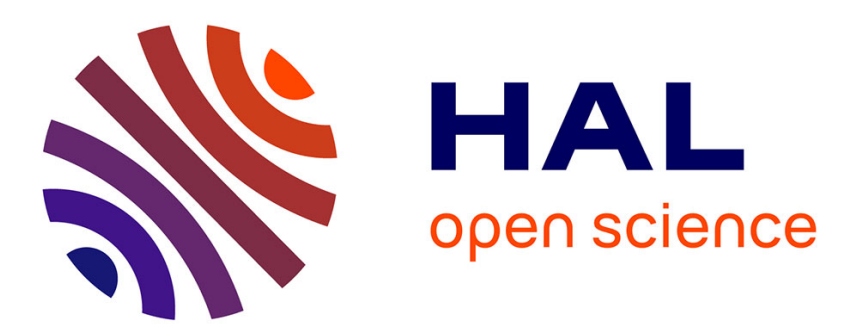

\title{
A Construction of Thermal Basis Functions for Coupled Structural - Thermal Reduced Order Models
}

\author{
Raghavendra Murthy, X.Q. Wang, Andrew Matney, Marc Mignolet
}

\section{To cite this version:}

Raghavendra Murthy, X.Q. Wang, Andrew Matney, Marc Mignolet. A Construction of Thermal Basis Functions for Coupled Structural - Thermal Reduced Order Models. 58th AIAA/ASCE/AHS/ASC Structures, Structural Dynamics, and Materials Conference, Jan 2017, Grapevine, United States. 10.2514/6.2017-0179 . hal-01712954

\section{HAL Id: hal-01712954 https://hal.science/hal-01712954}

Submitted on 26 Feb 2018

HAL is a multi-disciplinary open access archive for the deposit and dissemination of scientific research documents, whether they are published or not. The documents may come from teaching and research institutions in France or abroad, or from public or private research centers.
L'archive ouverte pluridisciplinaire HAL, est destinée au dépôt et à la diffusion de documents scientifiques de niveau recherche, publiés ou non, émanant des établissements d'enseignement et de recherche français ou étrangers, des laboratoires publics ou privés. 


\title{
A CONSTRUCTION OF THERMAL BASIS FUNCTIONS FOR COUPLED STRUCTURAL - THERMAL REDUCED ORDER MODELS
}

\author{
Raghavendra Murthy, X.Q. Wang, Andrew K. Matney, and Marc P. Mignolet \\ SEMTE, Faculties of Mechanical and Aerospace Engineering, Arizona State University, \\ 501 E. Tyler Mall, Tempe, Arizona, 85287-6106
}

\begin{abstract}
The focus of this paper is on the formulation and assessment of a novel approach for the construction of a thermal basis to be used in coupled nonlinear geometric structural-thermal reduced order modeling efforts. The intent is to have this basis rely as little as possible on the existence of snapshots of the temperature distribution obtained by full order computations, i.e., finite element and CFD solutions, to reduce the numerical effort associated with their determination. To this end, the core of the basis is first constructed from the recently introduced optimum thermal modes which are the temperature distributions that most strongly affect the structural response and thus are independent of the applied heat flux. It is shown that this basis is not sufficient and thus a limited number of enrichments are introduced that do come from such full order computations and which complete the basis. The approach is demonstrated on a representative hypersonic panel in fully coupled aero-structural-thermal interaction.
\end{abstract}

\section{INTRODUCTION}

Notwithstanding its rather long history, e.g., see [1-3] and references therein, the aerodynamic/structural/thermal analysis of hot structures remains particularly challenging. One key difficulty in carrying out predictions of the structural response and temperature distribution is the complex coupling between the various disciplines that may be present. While there is no direct feedback (neglecting the small latency effect) of structural deformations on temperature, Culler and McNamara [1] have demonstrated that there may be significant indirect feedback through changes in the aerodynamics that changes dramatically the temperature field. Since the level of this indirect feedback is not known in advance/is hard to estimate, it is concluded from [1] that a fully coupled fluid-structure-thermal analysis should be carried out for hypersonic vehicles.

Performing this analysis with standard full order models, i.e., finite element models to predict the structural deformations and the temperature and a computational fluid dynamics (CFD) code, is a daunting task computationally especially since these codes typically do not pass the information to each other efficiently as observed in [1]. Reduced order models however are much more computationally expedient but also permit an easier connectivity; they are thus the approach of choice for fluid-thermal-structure interaction (FTSI) problems such as those on hypersonic vehicles. The reduced order models (ROMs) to be employed should be derived from commercial (e.g., Nastran) finite element models of the structure considered to render them directly usable for practical applications. Moreover, they should include nonlinear geometric effects that arise when the structural deformations become "large".

The construction of such ROMs has been the subject of continuous focus in the last few years, e.g., see [4-7], that has firmed up the process. One issue which remains challenging is the 
selection of the basis functions to be used to represent the temperature distribution. While snapshots of temperature distribution obtained in full computations could successfully be used, e.g., see [2,3], it would be desirable to have the basis formed, at least in part, from a priori information. Proceeding in this manner would likely reduce the number of snapshots to be determined but also would provide robustness with respect to unexpected physics and/or a poor selection of the snapshots. This paper will propose such a basis and provide a first demonstration of it on the panel investigated in [1,5-7].

\section{COUPLED STRUCTURAL-THERMAL REDUCED ORDER MODELING}

The reduced order models considered here are based on a representation of the temperature and displacement fields in the expansion format

$$
\boldsymbol{T}(t)=\sum_{n=1}^{\mu} \tau_{n}(t) \boldsymbol{T}^{(n)} \quad \text { and } \quad \boldsymbol{u}(t)=\sum_{n=1}^{M} q_{n}(t) \Psi^{(n)}
$$

Here, $q_{n}(t)$ and $\tau_{n}(t)$ are the time-dependent structural and thermal generalized coordinates, while $\boldsymbol{T}^{(n)}$ and $\Psi^{(n)}$ are the structural and thermal basis functions, or modes. With the representation of Eqs (1) and (2), the governing equations for the structural and thermal generalized coordinates are (see $[4,8]$ for derivation, summation over repeated indices implied)

$$
\begin{gathered}
M_{i j} \ddot{q}_{j}+D_{i j} \dot{q}_{j}+K_{i j}^{(1)}\left(\tau_{r}\right) q_{j}+K_{i j l}^{(2)}\left(\tau_{r}\right) q_{j} q_{l}+K_{i j l p}^{(3)}\left(\tau_{r}\right) q_{j} q_{l} q_{p}=F_{i}\left(\tau_{r}\right) \\
B_{i j}\left(\tau_{l}\right) \dot{\tau}_{j}+\tilde{K}_{i j}\left(\tau_{l}\right) \tau_{j}=P_{i}
\end{gathered}
$$

where the mass matrix elements are denoted by $M_{i j}$, while the linear, quadratic, and cubic stiffness coefficients are $K_{i j}^{(1)}, K_{i j l}^{(2)}$, and $K_{i j l p}^{(3)}$, respectively. Further, $F_{i}$ denotes the generalized forces while $K_{i j l}^{(t h)}$ and $F_{i l}^{(t h)}$ provide the influence of thermal loading on the structural response, thereby coupling the thermal and structural problems. The symbols $B_{i j}$ and $\tilde{K}_{i j}$ represent the elements of the capacitance and conductance matrices. The heat convection with the fluid as well as the heat flux due to radiation are lumped in the source term, $P_{i}$.

Equations (3) and (4) hold whether the material has temperature dependent or temperature independent properties. In the latter case, only the linear stiffness terms $K_{i j}^{(1)}\left(\tau_{l}\right) q_{j}$ and the generalized forces $F_{i}\left(\tau_{l}\right)$ depend explicitly on the temperature generalized coordinates, and only linearly, i.e.,

$$
\begin{gathered}
K_{i j}^{(1)}\left(\tau_{l}\right) q_{j}=K_{i j}^{(1)} q_{j}-K_{i j l}^{(t h)} q_{j} \tau_{l} \\
F_{i}\left(\tau_{l}\right)=F_{i}+F_{i l}^{(t h)} \tau_{l} .
\end{gathered}
$$

When the elasticity tensor and the coefficient of thermal expansion depend linearly on local temperature, the linear stiffness and generalized forces become cubic functions of the temperature generalized coordinates, i.e.,

$$
K_{i j}^{(1)}\left(\tau_{l}\right)=K_{i j}^{(1)}-K_{i j l}^{(t h)} \tau_{l}+\hat{K}_{i j, l r}^{(t h)} \tau_{l} \tau_{r}+\tilde{K}_{i j, l r s}^{(t h)} \tau_{l} \tau_{r} \tau_{s}
$$

and 


$$
F_{i}\left(\tau_{l}\right)=F_{i}+F_{i l}^{(t h)} \tau_{l}+\hat{F}_{i l r}^{(t h)} \tau_{l} \tau_{r}+\tilde{F}_{i l r s}^{(t h)} \tau_{l} \tau_{r} \tau_{s}
$$

while the quadratic and cubic stiffness terms become linear functions of the temperature generalized coordinates, that is

$$
\begin{aligned}
& K_{i j l}^{(2)}\left(\tau_{l}\right)=K_{i j l}^{(2)}+\hat{K}_{i j l, r}^{(2)} \tau_{r} \\
& K_{i j l}^{(3)}\left(\tau_{l}\right)=K_{i j l}^{(3)}+\hat{K}_{i j l, r}^{(3)} \tau_{r} .
\end{aligned}
$$

Finally, if only the coefficient of thermal expansion is dependent on local temperature, and linearly so, the parameters $K_{i j l}^{(2)}$, and $K_{i j l p}^{(3)}$ are constant and the linear stiffness and generalized forces are only quadratic functions of the temperature generalized coordinates, i.e.,

$$
K_{i j}^{(1)}\left(\tau_{l}\right)=K_{i j}^{(1)}-K_{i j l}^{(t h)} \tau_{l}+\hat{K}_{i j, l r}^{(t h)} \tau_{l} \tau_{r}
$$

and

$$
F_{i}\left(\tau_{l}\right)=F_{i}+F_{i l}^{(t h)} \tau_{l}+\hat{F}_{i l r}^{(t h)} \tau_{l} \tau_{r}
$$

Having established the forms of the governing equations, i.e., Eqs (3) and (4), it remains to address (i) the selection of the basis functions $\boldsymbol{T}^{(n)}$ and $\Psi^{(n)}$ and (ii) the identification of all stiffness, mass, conductance, and capacitance parameters from commercial finite element software. The latter effort is detailed in [4,9-11] for temperature independent structural properties and has been extended in [4] in the linearly varying case. The former issue has also been addressed, in [10] for the structural problem, see also [9,11] for the linear + dual modes basis selected here. Finally, the construction of the thermal basis, which represents the focus of this investigation, is addressed in the next sections.

\section{THERMAL ROM BASIS CONSTRUCTION}

Given the above discussion, a key remaining issue in the thermal ROM modeling is the selection of reliable basis functions $T^{(n)}$. Specifically, it is proposed here that the thermal basis be composed of two components:

(1) a series of "optimum thermal modes" as defined in [7], i.e., temperature distributions that most strongly affect the structural ROM with temperature independent properties. As shown in [7], these modes are in fact derived from stress distributions associated with the structural modes selected, and

(2) enrichments which are obtained from the residuals of projection on the optimum thermal modes of the temperature distributions obtained from a small set of representative full order computations.

\section{Optimum Thermal Modes}

As shown in particular in Eq. (3), the temperature of the panel may (often does) strongly affect the structural response. This observation recently motivated [7] the search for the temperature distributions that have a maximum effect on this response with the intent that these distributions should be included in the thermal basis so that the thermal-structural coupling is well captured by this basis. This effort was conducted for temperature independent properties and thus the terms which must be maximized are 


$$
T E_{i j}^{(1)}=K_{i j l}^{(t h)} \tau_{l} \quad \text { and } \quad T E_{i}^{(2)}=F_{i l}^{(t h)} \tau_{l} .
$$

It was found [7] that the temperature distributions maximizing these quantities are the fields $G_{i j}$ and $H_{i}$, respectively, defined as

$$
G_{i j}=S_{i j, v r}^{N L}(\boldsymbol{X}) \alpha_{v r} \text { and } H_{i}=S_{i, v r}^{L}(\boldsymbol{X}) \alpha_{v r}
$$

where $S_{i, v r}^{L}(\boldsymbol{X})$ and $S_{i j, v r}^{N L}(\boldsymbol{X})$ denote the $v r$ components of the linear and nonlinear stress tensors at location $\boldsymbol{X}$ induced by the structural modes $i$ and $i$ and $j$, respectively. Moreover, $\alpha_{v r}$ denotes the $v r$ component of the coefficient of thermal expansion tensor.

Mapping the fields $G_{i j}(\boldsymbol{X})$ and $H_{i}(\boldsymbol{X})$ from the structural finite element model (on which the stresses are defined) to the thermal one results in vectors $\boldsymbol{G}_{i j}$ and $\boldsymbol{H}_{i}$ which are in general not allowable temperature distributions as they do not satisfy the thermal boundary conditions. They are thus modified as follows. First, the static fluxes on the structure $\phi_{i j}$ and $\phi_{i}$ that would give rise to $\boldsymbol{G}_{i j}$ and $\boldsymbol{H}_{i}$ are obtained by multiplying these vectors by the conductance matrix, i.e.,

$$
\phi_{i j}=\tilde{\boldsymbol{K}} \boldsymbol{G}_{i j} \quad \text { and } \quad \phi_{i}=\tilde{\boldsymbol{K}} \boldsymbol{H}_{i} .
$$

These fluxes are then reapplied to the structure where a nonzero flux is indeed consistent with the problem and with all other linear thermal boundary conditions, e.g., fixed temperature, adiabatic boundary conditions, satisfied. The radiation boundary conditions must be applied as well since their lack would often lead to diverging temperature distribution as time progresses. However, they cannot be applied directly as they are nonlinear and thus strongly dependent on the magnitude of the temperature profile which is yet unknown. The magnitudes are not correctly represented by the vectors $\phi_{i j}$ and $\phi_{i}$ which come from arbitrarily scaled stress distributions. Accordingly, the radiation is replaced by a linear boundary condition, i.e., a convection boundary condition to a medium at zero temperature. Current experience with this process seems to indicate that the form of the resulting temperature distribution, which is what is required for the basis functions sought here, is slightly dependent on the value of the coefficient of convection selected, i.e., choosing a coefficient of convection that leads to convective heat transfer of approximately the same order of magnitude as that which results from the applied heat fluxes leads to a more representative temperature distribution. In the absence of such information, these computations can also be repeated with several values of the coefficient of convection and the set of temperature distributions could be used in the ensuing POD analysis as opposed to a single distribution.

This process leads to tentative temperature basis functions $\boldsymbol{T}_{\mathbf{i j}}{ }^{\mathbf{L}}$ and $\boldsymbol{T}_{\boldsymbol{i}}{ }^{\boldsymbol{L}}$ which are referred to as enhanced stress distributions and there will be one of each for each value of $i$ and $j$ spanning the ensemble of structural modes. This set of distributions would form a basis much larger than what is desired and the final step of the process is accordingly to reduce them to the most significant ones. To this end, the coefficients $K_{i j l}^{(t h)}$ are computed for each function $\boldsymbol{T}_{\mathbf{i j}}{ }^{\boldsymbol{N}}$ (see [7] for discussion) to form the matrix $\hat{\boldsymbol{V}}$ of components 


$$
\hat{V}_{I l}=\frac{K_{i j l}^{(t h)}}{\sqrt{K_{i i}^{(1)} K_{j j}^{(1)}}} .
$$

A proper orthogonal decomposition (POD) analysis is next carried out and the eigenvectors corresponding to the largest eigenvalues are identified; they provide the linear combinations of the functions $T_{i j}^{N L}$ that lead to the largest overall effect on the structural response starting from the nonlinear stress distributions. A similar POD analysis is also carried out using the matrix

$$
V_{i l}=F_{i l}^{(t h)} \text {. }
$$

where the thermal generalized forces $F_{i l}^{(t h)}$ are determined from the functions $\boldsymbol{T}_{\boldsymbol{i}}^{\boldsymbol{L}}$. Assembling the two sets of dominant eigenvectors finally produces the desired optimum thermal mode component of the thermal basis.

The enhancement of the stress distributions described above is based on steady thermal computations and is thus appropriate when the structure is reaching such a state. Given the slow time scale of the heat diffusion, it is reasonable to expect that transient thermal effects may take place, e.g., during ascend and descend, see the validation example below. In such conditions, using steady state data will not provide all the critical information for the temperature modeling. For example, the steady state temperature distribution of a thin panel heated on one side and exhibiting an adiabatic boundary condition on the other is essentially constant through thickness. This is in contrast with the transient situation where a difference between top and bottom temperature may be observed that plays an important role in inducing structural deformations. To capture transient thermal information, a limited set of transient thermal analyses are suggested that exactly parallel the enhancement described above, i.e., the constant fluxes $\phi_{i j}$ and $\phi_{i}$ are applied to the appropriate part of the structure from $t=0$ starting from a uniform zero temperature structure and with the same boundary conditions as described above. Snapshots of the temperature distributions in the beginning of this time marching can then be collected and a POD analysis carried out to extract the dominant features of their residuals of projection on the (steady) optimum thermal modes. The POD eigenvectors retained to complement the basis will be referred to as transient optimum thermal modes.

\section{Thermal Modes Enrichments}

The steady and transient optimum thermal modes described above capture the temperature effects on the structure reasonably well. However, they do not account for peculiarities of the thermal problem, such as actual heat flux distribution, variation with temperature of conductance/capacitance, etc. Hence, additional basis functions referred to as thermal enrichments modes will be necessary to complement the basis and those must originate from a limited set of full order solutions. Specifically, these enrichments are constructed by carrying out a proper orthogonal decomposition on the residuals of projection on the steady and transient optimum thermal modes of temperature snapshots resulting from coupled fluid-thermalstructural analyses with exact boundary conditions, e.g., radiation included, temperature dependent thermal properties as appropriate, etc. Examples of snapshots used for the determination of these enrichments will be presented in connection with the validation example. 


\section{VALIDATION EXAMPLE: A REPRESENTATIVE HYPERSONIC PANEL Structural-Thermal-Aerodynamic Model}

The representative hypersonic panel of Fig. 1 was considered next as a basis for validating the construction strategy discussed. Its structural finite element model [1] is composed of 2400 CQUAD4 (4 node plate) elements, resulting in 2499 nodes. In both the structural and thermal problems the dimensions of the element were $0.25 \mathrm{in}$. by $0.25 \mathrm{in}$. The panel has the following structural boundary conditions

(1) At the leading edge, $x=0$ and $z=0$, zero displacements are enforced for all degrees of freedom but $y$ translations, which are free.

(2) At the trailing edge, $x=12$ in and $z=0$, zero displacements are enforced for all degrees of freedom except $x$ and $y$ translations, which are free. Springs also act on the nodes of the trailing edge in the $x$ direction with a spring constant of $2378 \mathrm{lb} / \mathrm{in}$.

(3) At the panel center, $x=6$ in, $y=5$ in, and $z=0$, zero displacement is enforced for y translations.

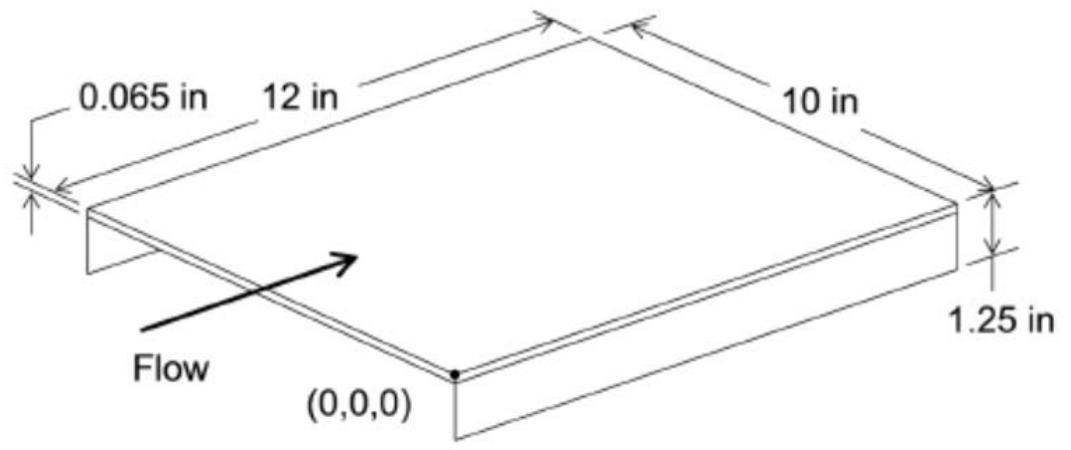

Figure 1. Representative Hypersonic Panel

The structural properties of the composite material used in this model, advanced carbon-carbon 4, are provided in Tables 1 [1]. These properties, except for the coefficient of thermal expansion will remain constant since there is no significant change over the range of temperatures experienced in this analysis. Moreover, the coefficient of thermal expansion was assumed to vary linearly between the data given in Table 2 .

The thermal finite element model [1] consisted of 8-node brick elements (CHEXA) on the skin and plane elements (CQUAD4) on the stiffeners for a total to 2400 elements and 4508 nodes. The CHEXA elements allowed for the through thickness temperature gradient to be calculated while the CQUAD4 elements yielded a constant temperature through thickness. This choice of elements resulted from the boundary conditions which were adiabatic on the stiffeners and the bottom of the skin while on its top a flux boundary condition was imposed accounting for both convection and radiation. With these boundary conditions, a near constant temperature through the thickness of the stiffeners could be expected while a variation of temperature through the skin thickness would take place. Since the structural elements on the skin permit only the imposition of a linear temperature profile, a single thermal element through the thickness of the skin was adopted. The thermal properties of the panel are defined as a function of temperature in Tables 3 except for the emissivity $(=0.8)$ and the $(3,3)$ component of the thermal conductivity (=3.0 BTU $\left./ \mathrm{hr} / \mathrm{ft} /{ }^{\circ} \mathrm{F}\right)$. 
Table 2. Material Properties of Panel

\begin{tabular}{|c|c|l|c|c|c|}
\hline $\begin{array}{c}\text { Young's Moduli } \\
(\mathrm{psi})\end{array}$ & $1510^{6}$ & Density $\left(\mathrm{lbm} / \mathrm{in}^{3}\right)$ & 0.065 & $\begin{array}{c}\text { Coeff. Therm. Exp. } \\
\left(1 /{ }^{\circ} \mathrm{F}\right) \text { at } 30^{\circ} \mathrm{F}\end{array}$ & $0.55610^{-6}$ \\
\hline Shear Modulus $_{12}$ & $\begin{array}{c}2.5 \\
10^{6}\end{array}$ & Poisson's Ratio $_{12}$ & 0.30 & $\begin{array}{c}\text { Coeff. Therm. Exp. } \\
\left(1 /{ }^{\circ} \mathrm{F}\right) \text { at } 2500^{\circ} \mathrm{F}\end{array}$ & $2.33410^{-6}$ \\
\hline
\end{tabular}

Table 3. Specific Heat (c) and In-plane Conductivity ( $k$ ) vs. Temperature (T)

\begin{tabular}{|c|c|c|c|c|c|}
\hline$T\left({ }^{\circ} \mathrm{F}\right)$ & $c\left(\mathrm{BTU} / \mathrm{bm} /{ }^{\circ} \mathrm{F}\right)$ & $k\left(\mathrm{BTU} / \mathrm{hr} / \mathrm{ft} /{ }^{\circ} \mathrm{F}\right)$ & $T\left({ }^{\circ} \mathrm{F}\right)$ & $c\left(\mathrm{BTU} / \mathrm{lbm} /{ }^{\circ} \mathrm{F}\right)$ & $k\left(\mathrm{BTU} / \mathrm{hr} / \mathrm{ft} /{ }^{\circ} \mathrm{F}\right)$ \\
\hline 0 & 0.170 & 17.5 & 1500 & 0.330 & 23.3 \\
\hline 200 & -- & 20.9 & 1750 & - & 23.1 \\
\hline 500 & 0.242 & 23.6 & 2000 & 0.360 & 22.5 \\
\hline 750 & - & 24.2 & 2250 & - & 21.9 \\
\hline 1000 & 0.295 & 24.2 & 2500 & 0.390 & 21.4 \\
\hline 1250 & - & 23.9 & 2750 & - & 20.9 \\
\hline
\end{tabular}

Finally, the aerodynamic pressure and aerodynamic heating was modeled using piston theory and Eckert's reference enthalpy method, respectively. The vehicle was accelerated from Mach 2 to Mach 12 over 300 seconds, while the dynamic pressure was held constant at 2,000 psf. The structural, thermal and aerodynamic solutions were marched in time in a process described in detail in [1]. One-way coupling refers to the analysis in which the thermal problem is executed independently of the structural deformation. The temperature fields are fed to the structural solver and influence its response, but the structural displacement does not affect the heating on the panel. Two-way coupling refers to analyses in which the heating on the panel is influenced by the structural displacement. It is this latter format that more closely resembles reality, and is the subject of the work presented here.

It should be noted that the development of an accurate ROM of the two-way coupled situation was achieved through a series of steps in [5,6, and references therein] which serves as baseline for the present investigation, the 44 structural modes and the thermal modes and the corresponding generalized coordinates time histories more specifically.

\section{Optimum Thermal Modes}

Following the methodology presented in [7], 44 optimum thermal modes from the linear stress distributions and 741 from the nonlinear ones were first selected. They were then modified as discussed above with the heated part of the panel being only the top of the skin. Shown in Figs 2 and 3 are typical examples of the linear and nonlinear stress distributions before and after the enhancement process.

A final contraction of that ensemble of possible temperature distributions was carried out using the POD analysis of Eq. (18), and all 44 linear and the first 20 nonlinear POD modes were selected as the steady optimum thermal basis based on the decay of the POD eigenvalues as shown in Fig. 4. A uniform temperature mode was then added to account directly for the overall heating of the panel. As shown in Fig. 5 and 6, this 65 mode basis provides a very good representation of the temperature distribution on the top of the skin but not on the stiffeners 


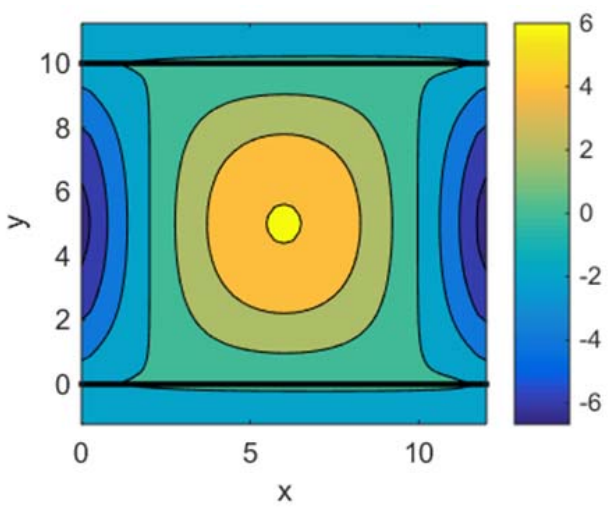

(a)

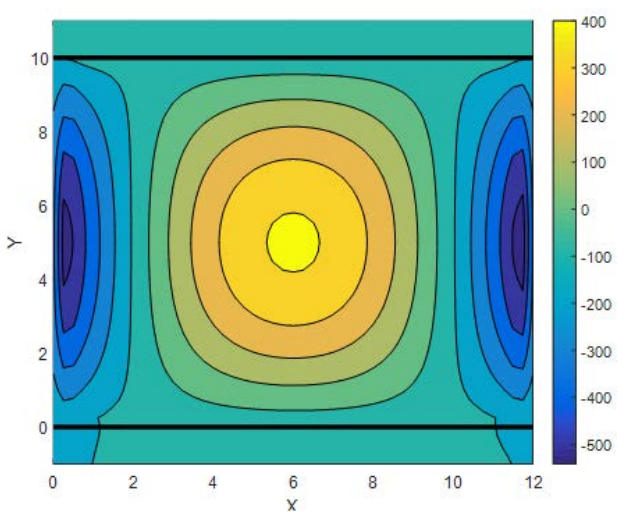

(b)

Figure 2. Linear Stress Distribution Corresponding to Structural Mode 1

(a) Original and (b) Enhanced.

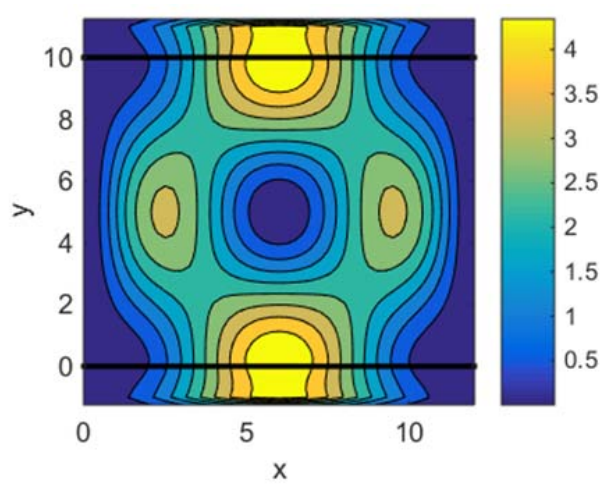

(a)

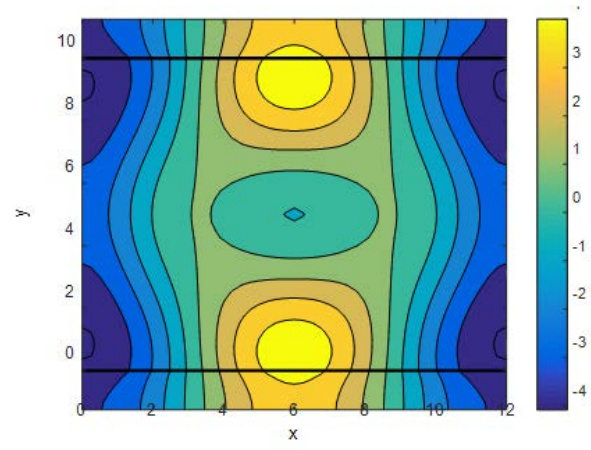

(b)

Figure 3. Nonlinear Stress Distribution Corresponding to Structural Mode 1

(a) Original and (b) Enhanced.

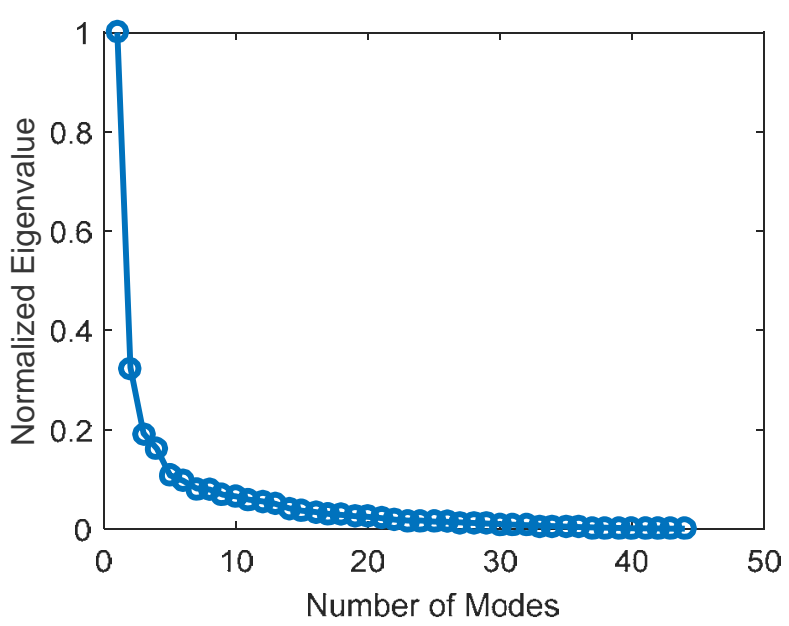

(a)

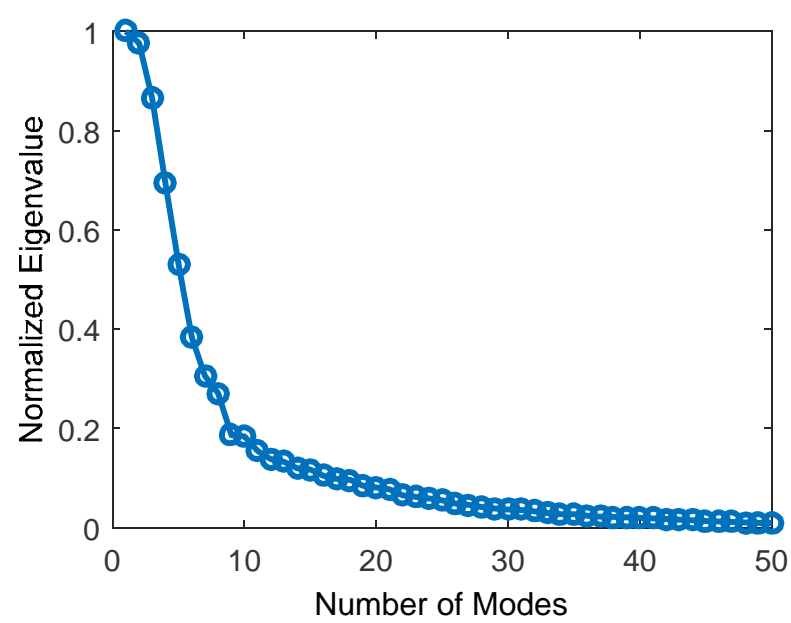

(b)

Figure 4. POD Eigenvalues of (a) Enhanced Linear Stress Distributions and (b) Enhanced Nonlinear Stress Distributions. 


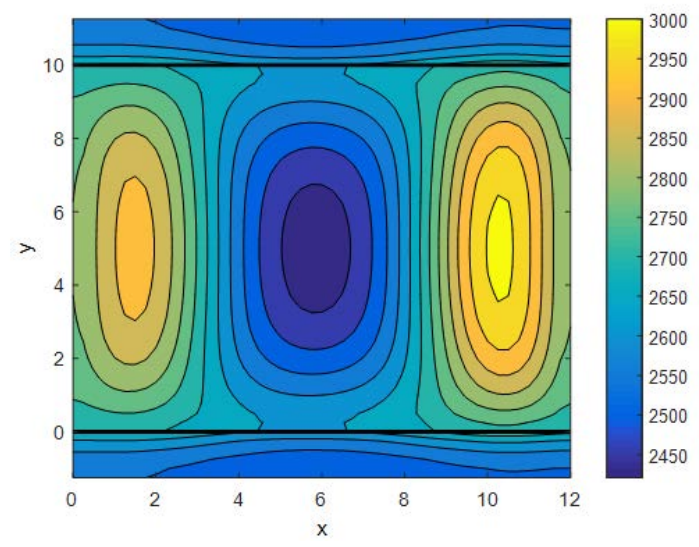

Figure 5. Temperature Baseline Data from [1].

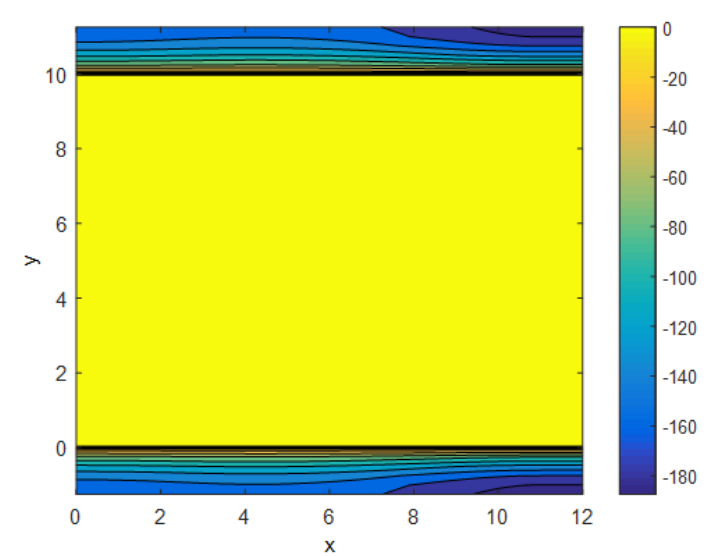

Figure 6. Temperature Representation Error Using the Optimum Thermal Modes Only

As stated above, the panel is undergoing a significant acceleration and thus it may be expected that transient thermal effects can be observed. This is indeed the case and a difference of temperature between top and bottom of the skin was observed (e.g., see [5,6]). A detailed analysis of the representation error (see Fig. 6) demonstrated as well that this difference was not captured with the steady optimum thermal modes. Accordingly, transient thermal analyses were carried out by applying the constant heat fluxes $\phi_{i}$ corresponding to the first two dominant structural modes starting at $t=0$ with a uniform zero temperature distribution of the panel. The first 0.1 seconds of the transient thermal solution temperature distribution snapshots were determined and were projected onto the steady optimum thermal modes and the residuals computed. The coefficients $F_{i l}^{(t h)}$ were computed for each of these residuals to form the matrix $\hat{\boldsymbol{V}}$, see Eq. (18), and the POD eigenvectors corresponding to the largest POD eigenvalues of $\hat{\boldsymbol{V}}$ were included as addition to the steady optimum thermal modes. Analyzing the decay of the POD eigenvalues, see Fig. 7, let to the selection of 6 such additions (i.e., transient optimum thermal modes), 3 from the first dominant structural mode, and 3 from the second dominant structural mode.

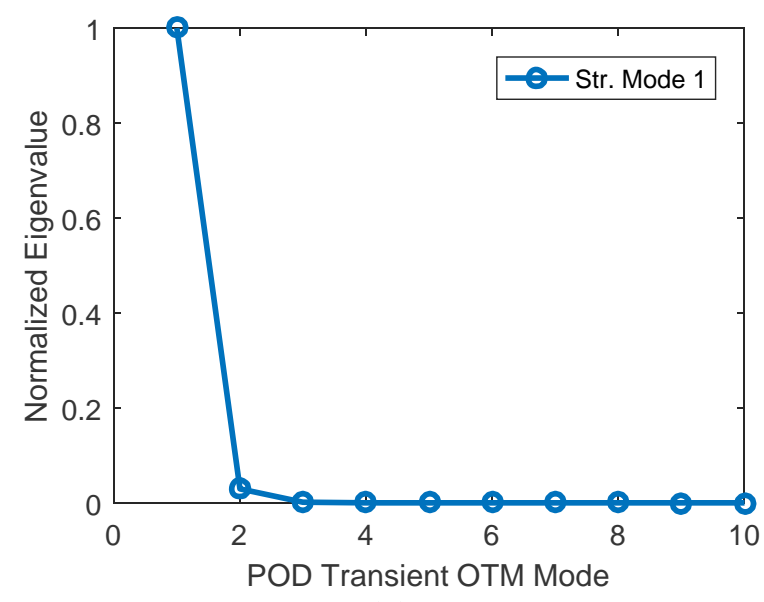

(a)

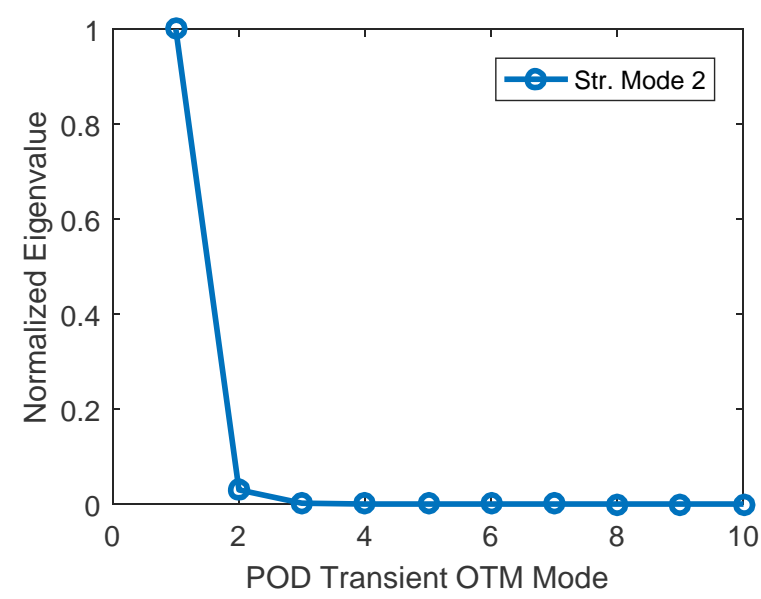

(b)

Figure 7. POD Eigenvalues of Residuals for Transient Optimum Thermal Modes for

(a) Structural Mode 1 and (b) Structural Mode 2 
Thermal enrichments were finally considered to complete the thermal basis. To generate the snapshots on which those enrichments are based, the panel was deformed statically along each linear structural mode in the structural basis, appropriately scaled. A series of deformation scaling factors were explored ranging from small linear deformations (with a maximum deformation of half of the panel thickness) to the limit of expected large nonlinear deformations (with a deformation of three times the panel thickness). Deformations both into and out of the flow were considered at a series of Mach numbers (Mach 2 to Mach 12) at which the panel was expected to operate. At each static deformation of the panel, the aerodynamic pressure and heating resulting from the deformation was computed using third order Piston Theory and Eckert's Reference Enthalpy method as in [1]. The computed convective heat flux was then applied on the panel top surface with appropriate boundary conditions (see [1]) and the first 20 seconds of the transient thermal solution temperature distribution snapshots were obtained. These snapshots were then projected onto the steady and transient optimum thermal basis and components of the snapshots from the optimum thermal basis space were removed to get residuals. The coefficients $F_{i l}^{(t h)}$ were computed for each of these residuals to form the matrix $\hat{\boldsymbol{V}}$, see Eq. (18), and the POD eigenvectors corresponding to the largest POD eigenvalues of $\hat{\boldsymbol{V}}$ were included as enrichments to the optimum thermal modes. A total of 9 enrichments, based on the decay of the POD eigenvalues, see Fig. 8, were added from to the optimum thermal modes to complete the thermal basis.

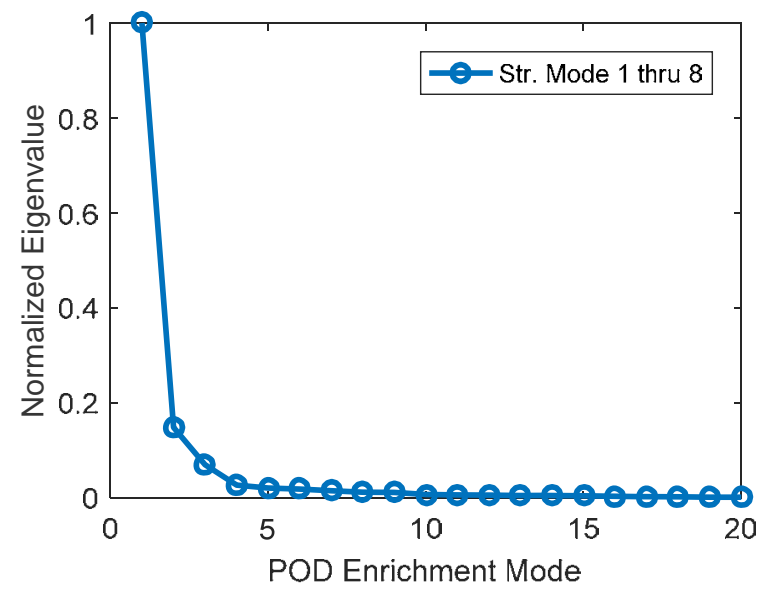

Figure 8. POD Eigenvalues of Residuals for Enrichment Modes

The above enrichments were determined from a POD analysis of the matrix $\hat{\boldsymbol{V}}$, i.e., they can be construed as the enrichments necessary to improve the effects of the temperature distribution on the structural deformations. However, the temperature modeling per se also needs to be improved. To this end, part of the above snapshots, i.e., those corresponding to the combination of structural modes 1 and 2 at all Mach numbers for a panel deformation levels of 2 and 3 thicknesses into and out of the flow, were projected directly (without passing through the matrix $\hat{\boldsymbol{V}}$ ) on the current temperature basis and a final POD analysis, of those residuals, was carried out. Shown in Figs 9 and 10 vs. the number of POD eigenvectors are the POD eigenvalues as well as the root mean squared temperature error over the entire panel for the final time-step of the coupled structural-thermal simulation. It can be observed that there is a very quick drop in the POD eigenvalues as well as the temperature representation error with increasing number of these 
last enrichment modes. In fact, with 5 POD modes considered, the root mean squared temperature error over the entire panel falls to within 1 degree $\mathrm{F}$.

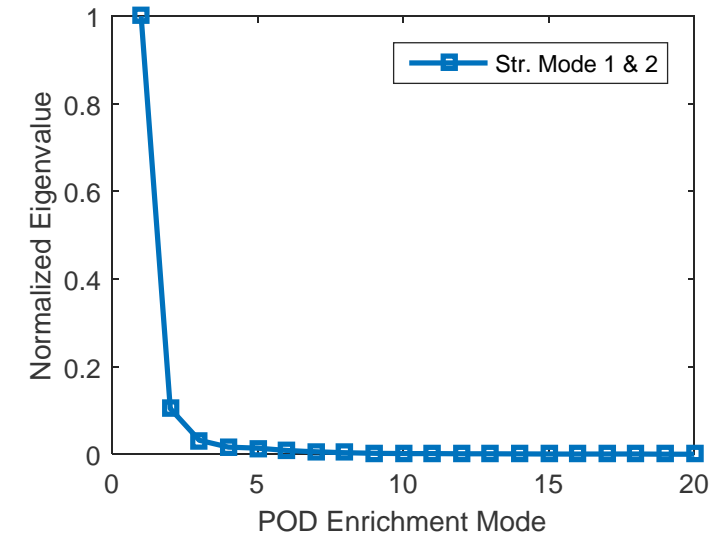

Figure 9. POD Eigenvalues of Residuals of Transient Temperature Snapshots

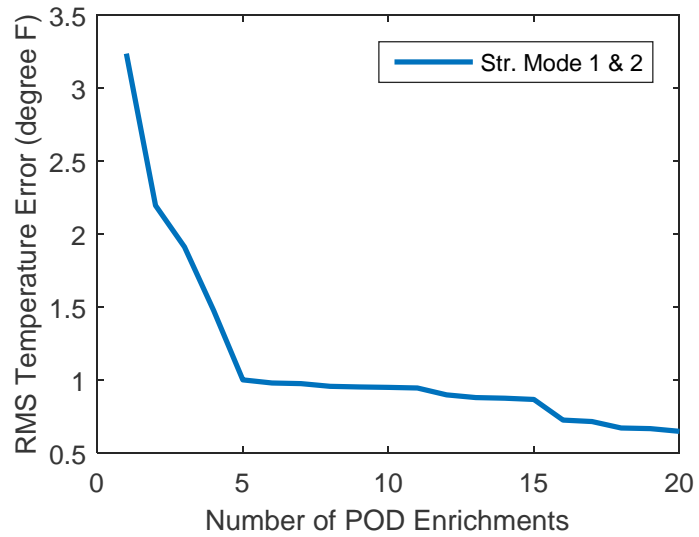

Figure 10. RMS Temperature Error vs. Number of POD Eigenvectors Kept in Addition to Optimum Thermal Modes

With these final 5 modes, the thermal basis is composed of 85 basis functions: 20+44 from the steady optimum thermal modes, the uniform temperature distribution, 6 transient optimum thermal modes, 9 enrichments of the temperature distribution effects on the structure, and 5 enrichments of the temperature modeling. Note that 71 of these modes are dependent on the structural model while only 14 result from full order computation snapshots all of which are on a statically deformed panel so that they do not involve full FTSI, only aerodynamic + thermal computations.

\section{Validation of the Thermal Basis}

A first assessment of the thermal basis was performed by projecting the time dependent temperature distribution obtained in [5,6] on the above 85-mode basis and comparing this projection with the original distribution. Shown in Fig. 11 is such a comparison for the final time step in the computation. The agreement is clearly very close. A similar quality comparison is shown in Fig. 12 for the time dependent temperature at points of the panel.

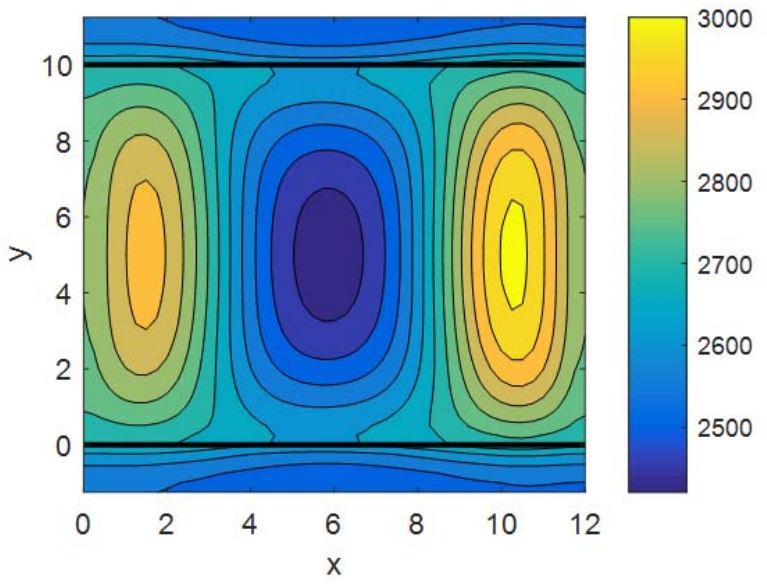

(a)

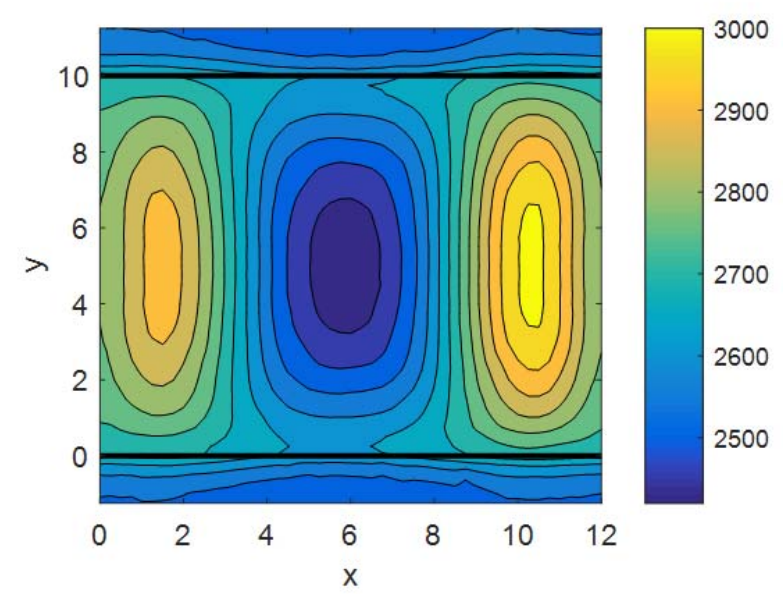

(b)

Figure 11. Comparison of (a) Baseline Temperature Distribution from [1] and (b) Reconstructed Temperature Distribution by Projection onto Thermal Basis 
Shown in Fig. 13 and 14 are comparisons of the temperature induced terms $F_{i l}^{(t h)} \tau_{l}$ and $K_{i j l}^{(t h)} \tau_{l}$ for the first few structural modes predicted from the baseline results of $[5,6]$ and from the projection of the temperature distribution on the 85-mode model. Once again, the agreement is excellent supporting the accuracy of this thermal basis.

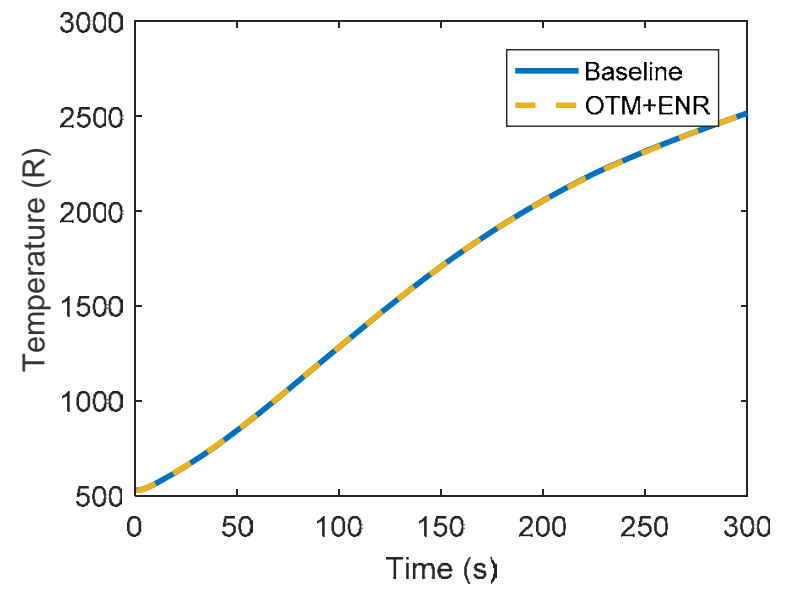

(a)

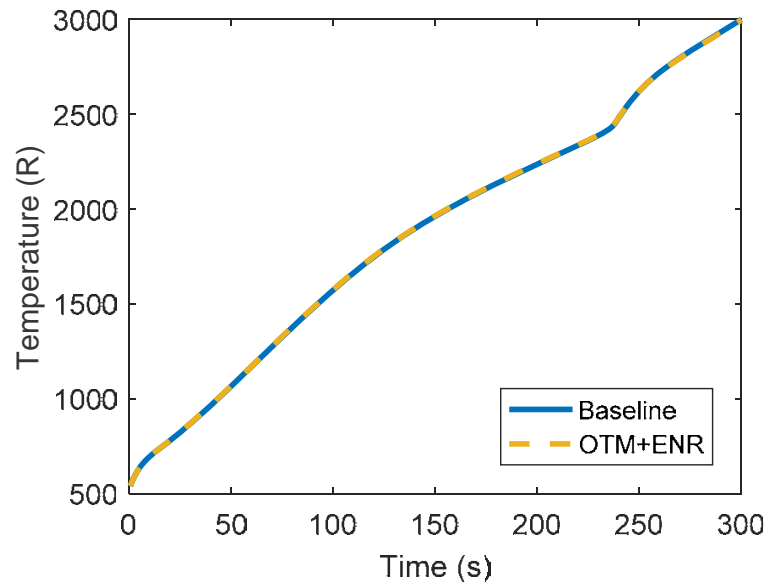

(b)

Figure 12. Pointwise Comparison of Temperature Evolution on (a) Stiffener, and (b) Skin of the Panel

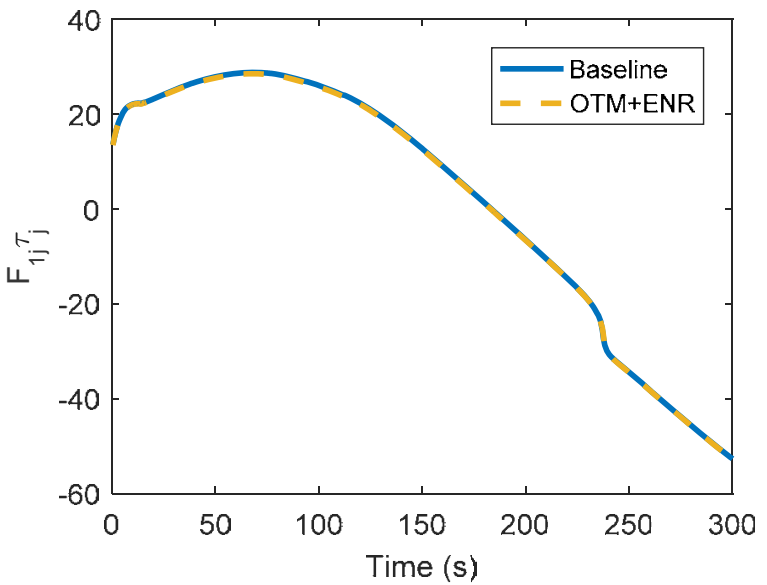

(a)

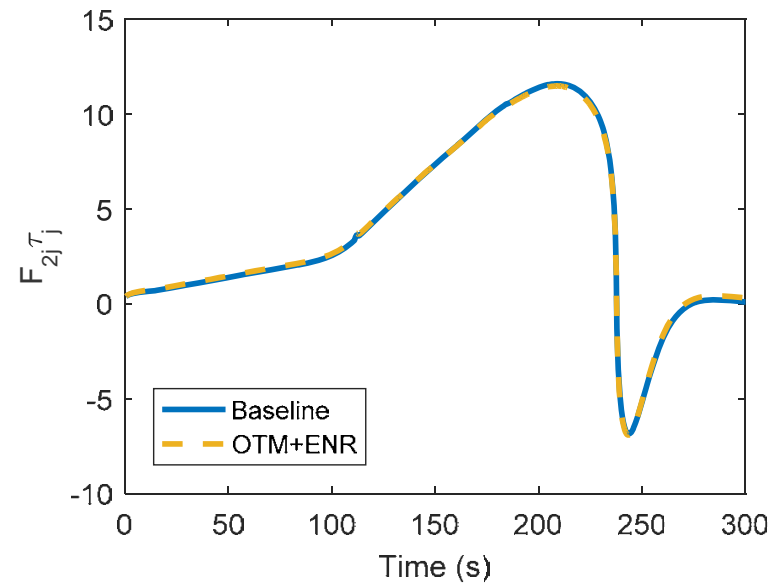

(b)

Figure 13. Comparison of the thermal loading terms $F_{i l}^{(t h)} \tau_{l}$ for structural mode (a) 1 and (b) 2 from [1] and from the Reconstructed Temperature Distribution by Projection onto Thermal Basis

\section{SUMMARY}

The focus of this paper was on the formulation and assessment of a novel approach for the construction of a thermal basis to be used in coupled nonlinear geometric structural-thermal reduced order modeling efforts. The first component of this basis are the recently introduced optimum thermal modes which are derived from the linear and nonlinear stress distributions induced by the structural modes. To conform with the thermal boundary conditions, these stress 


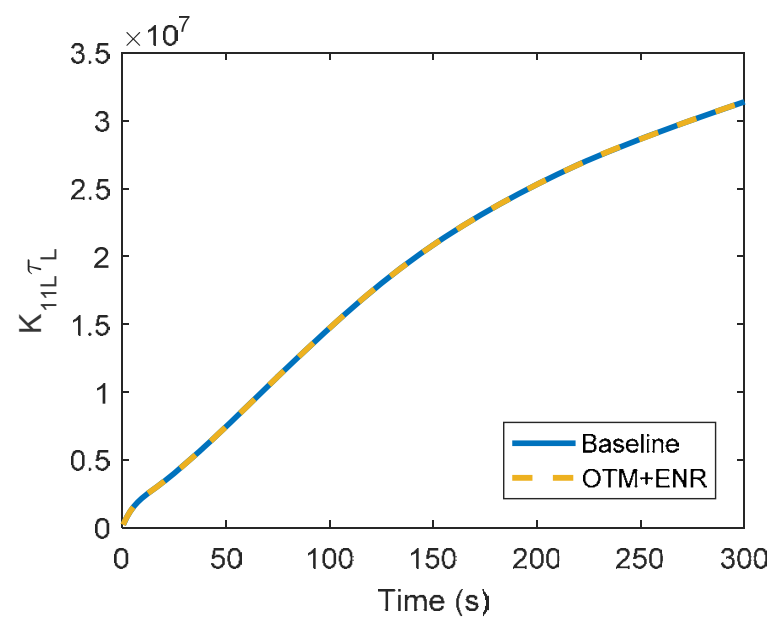

(a)

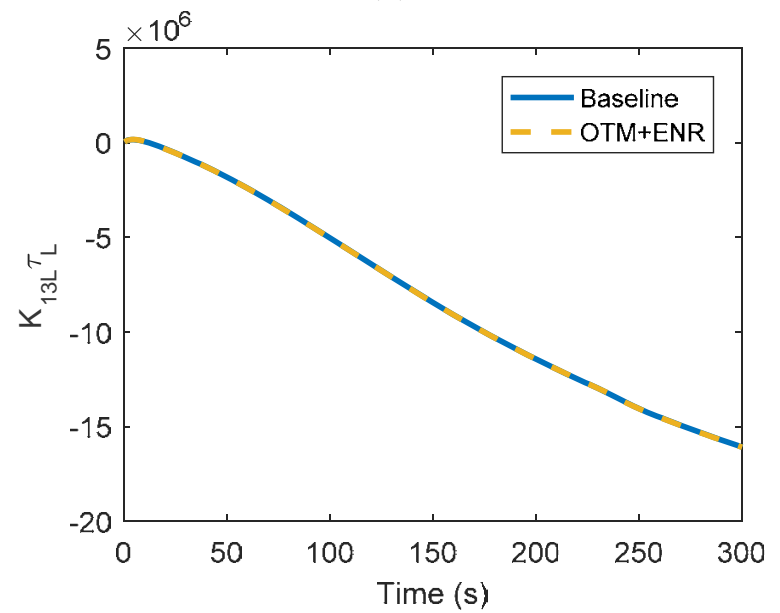

(c)

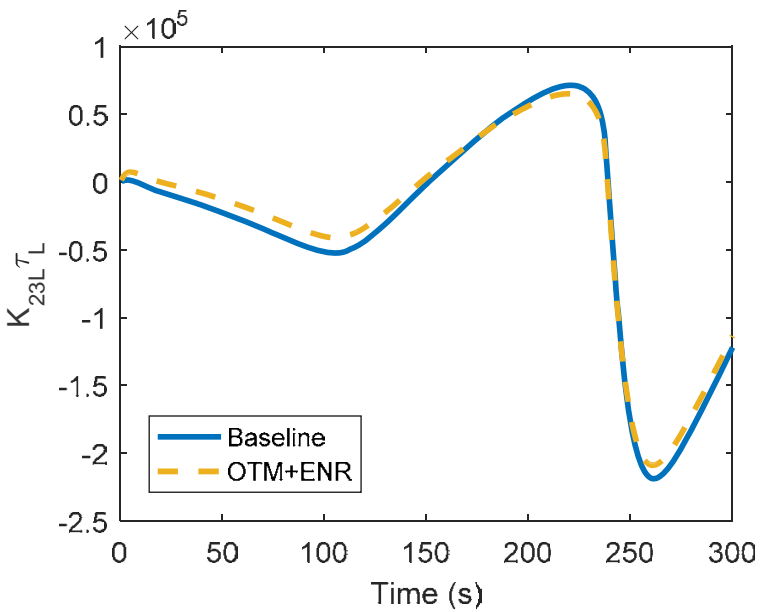

(e)

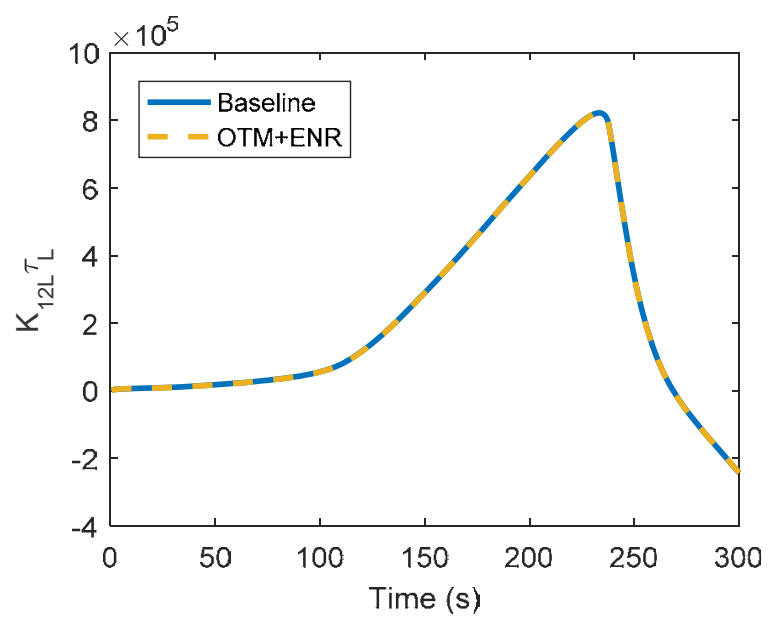

(b)

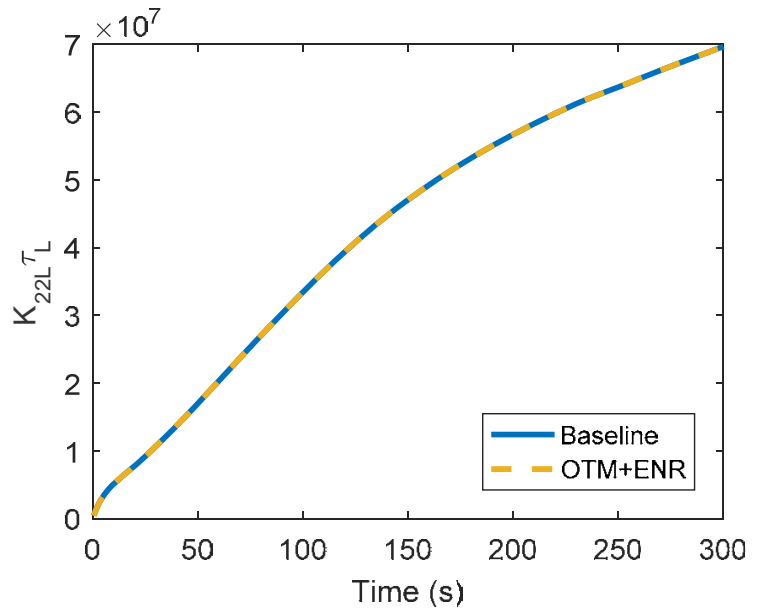

(d)

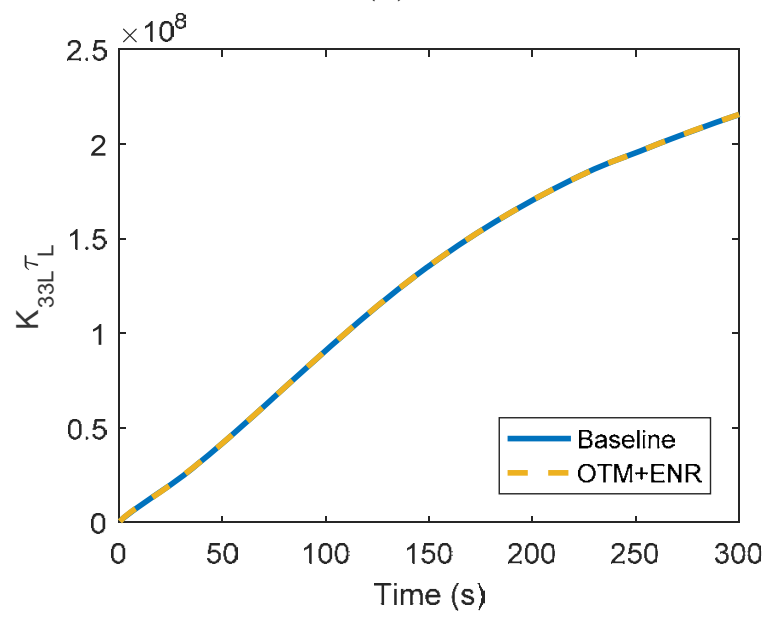

(f)

Figure 14. Comparison of the temperature induced terms $K_{i j l}^{(t h)} \tau_{l}$ for the first few structural modes from [1] and from the Reconstructed Temperature Distribution by Projection onto Thermal Basis 
distributions were first enhanced and then a POD analysis of them was carried out to reduce the size of this part of the basis. These modes are referred to as steady optimum thermal modes because they are enhanced through a steady thermal analysis and thus may not capture transient effects in the temperature distribution. To this end, the enhancement process can also be performed on short duration transient computations leading similarly to additional transient optimum thermal modes. Although these structure-driven thermal basis functions can provide a reasonable matching of actual temperature distributions, they need to be enriched for accurate computations. This enrichment is carried out by using full order computations of the thermal problem, or potentially the aero-structural-thermal coupled problem.

These concepts were demonstrated on a representative hypersonic panel for which full order and reduced order computations are already available. It is found that the basis provides a very good representation of the temperature and of its effects on the structure.

An additional supporting validation of this basis construction on a different panel is presented in [12].

\section{REFERENCES}

[1] Culler, A.J., and McNamara, J.J., "Impact of Fluid-Thermal-Structural Coupling on Response Prediction of Hypersonic Skin Panels,” AIAA Journal, Vol. 49, No. 11, 2011, pp. 2393-2406.

[2] N.J. Falkiewicz, C.E.S. Cesnik, "Proper Orthogonal Decomposition for Reduced-Order Thermal Solution in Hypersonic Aerothermoelastic Simulations,” AIAA Journal, Vol. 49, No. 5, 2011.

[3] N.J. Falkiewicz, C.E.S. Cesnik, A.R. Crowell, J.J. McNamara, "Reduced Order Aerothermoelastic Framework for Hypersonic Vehicle Control Simulation”, AIAA Journal, Vol. 49, No. 8, August 2011.

[4] R. Perez, X.Q. Wang and M.P. Mignolet, Nonlinear reduced order models for thermoelastodynamic response of isotropic and FGM panels. AIAA Journal, Vol. 49, pp. 630641, 2011.

[5] Matney, A.K., Mignolet, M.P., Spottswood, S.M., Culler, A.J., McNamara, J.J., "Thermal Reduced Order Model Adaptation to Aero-Thermo-Structural Interactions,” Proceedings of the AIAA Science and Technology Forum and Exposition (SciTech2014), National Harbor, Maryland, Jan. 13-17, 2014, Paper AIAA 2014-0493.

[6] Matney, A., Mignolet, M.P., Culler, A.J., McNamara, J.J., and Spottswood, S.M., "Panel Response Prediction through Reduced Order Models with Application to Hypersonic Aircraft," Proceedings of the AIAA Science and Technology Forum and Exposition (SciTech2015), Orlando, Florida, Jan. 5-9, 2015, AIAA Paper AIAA 2015-1630.

[7] Murthy, R., Wang, X.Q., Matney, A., and Mignolet, M.P., “Optimum Thermal Modes for Coupled Structural - Thermal Reduced Order Models,” Proceedings of the AIAA Science and Technology Forum and Exposition (SciTech2016), San Diego, California, Jan. 4-8, 2016, AIAA Paper AIAA 2016-1709.

[8] A.K. Matney, R.A. Perez and M.P. Mignolet, "Nonlinear Unsteady Thermoelastodynamic response of a Panel Subjected to an Oscillating Flux by Reduced Order Models," Proceedings of the 52nd Structures, Structural Dynamics and Materials Conference, Denver, Colorado, Apr. 4-7, AIAA 2011-2016, 2011. 
[9] K. Kim, A.G. Radu, X.Q. Wang and M.P. Mignolet, Nonlinear reduced order modeling of isotropic and functionally graded plates. International Journal of Non-Linear Mechanics, Vol. 49, pp. 100-110, 2013.

[10] M.P. Mignolet, A. Przekop, S.A. Rizzi and S.M. Spottswood. A review of indirect/nonintrusive reduced order modeling of nonlinear geometric structures. Journal of Sound and Vibration. Vol. 332, pp. 2437-2460, 2013.

[11] Perez, R.A., Wang, X.Q., and Mignolet, M.P., "Non-Intrusive Structural Dynamic Reduced Order Modeling for Large Deformations: Enhancements for Complex Structures," Journal of Computational and Nonlinear Dynamics, Vol. 9, No. 3, pp. 031008-1 - 031008-12, 2014.

[12] Gogulapati, A., Brouwer, K., Wang, X.Q. , Murthy R., McNamara, J.J., and Mignolet, M.P., "Full and Reduced Order Aerothermoelastic Modeling of Built-Up Aerospace Panels in High-Speed Flows," Proceedings of the AIAA Science and Technology Forum and Exposition (SciTech2017), Grapevine, Texas, Jan. 9-13, 2017. 